\title{
Validación del Autoconcepto Forma 5 en Universitarios Peruanos: Una Herramienta para la Psicología Positiva
}

\author{
Validation of the Self-Concept Form 5 in Peruvian \\ Undergraduates: A Tool for Positive Psychology
}

\author{
Varinia Bustos*, a Amparo Oliver ${ }^{\mathrm{b}} \&$ Laura Galiana $^{\mathrm{b}}$ \\ ${ }^{a}$ Universidad ESAN, Lima, Perú \& ${ }^{b}$ Universidad de Valencia, Valencia, España
}

\begin{abstract}
Resumen
El autoconcepto, aunque central en la psicología tradicional, ha cobrado importancia recientemente en el contexto de la psicología positiva. El Autoconcepto Forma 5 (AF5) es una medida muy utilizada en el contexto hispanohablante. Sin embargo, no existen estudios sobre sus propiedades psicométricas más allá de la fiabilidad en población peruana. El objetivo es presentar las propiedades psicométricas de la escala multidimensional AF5 en universitarios peruanos. Se recogió una muestra de 527 estudiantes universitarios. La encuesta incluyó, además del AF5, medidas de esperanza disposicional y satisfacción con la vida. Se llevó a cabo un análisis factorial confirmatorio para evaluar la validez criterial, con resultados adecuados, $\chi^{2}(395)=1484.46, p<.01$, CFI $=.93$, RMSEA $=.07$. También se calcularon alfas de Cronbach (que oscilaron entre .73 y .82), así como indicadores de fiabilidad de los ítems. Finalmente, se correlacionaron las dimensiones del autoconcepto con la esperanza y la satisfacción con la vida, siendo todas las relaciones positivas y estadísticamente significativas. Los resultados ofrecen evidencia de adecuadas propiedades psicométricas de la AF5 en estudiantes peruanos, permitiendo a futuras investigaciones en este contexto ahondar más en el nuevo dibujo que ha supuesto la psicología positiva dentro de la psicología tradicional.

Palabras clave: Satisfacción con la vida, esperanza, análisis factorial confirmatorio, estudiantes, autoconcepto.
\end{abstract}

\begin{abstract}
Self-concept, although central to traditional psychology, has recently gained importance in the context of positive psychology. The Self-concept Form 5 (AF5) is one of the most used measures when it comes to the Spanish speaking context. However, there are no studies on its psychometric properties going beyond reliability in Peruvian populations. The aim is to present the psychometric properties of the AF5 multidimensional scale in Peruvian university students. A sample of 527 university students was recruited. The survey included, besides the AF5, measures of dispositional hope and life satisfaction. A confirmatory factor analysis was implemented, with adequate results, $\chi^{2}(395)=$ $1484.46, p<.01, \mathrm{CFI}=.93$, RMSEA $=.07$. Cronbach's alphas were also calculated (ranging from .73 to .82 ), as well as item reliability indicators. Finally, self-concept dimensions were correlated with hope and life satisfaction, and all of them showed to be positive and statistically significant. Results offer evidence of appropriate psychometric properties of the AF5 in Peruvian undergraduates, allowing future research in this context to deepen into the new picture that has drawn positive psychology into traditional psychology.

Keywords: Life satisfaction, hope, confirmatory factor analysis, undergraduate students, self-concept.
\end{abstract}

El autoconcepto es uno de los conceptos psicológicos centrales desde prácticamente el inicio de la psicología científica (James, 1890), siendo definido como la autopercepción del individuo (Shavelson, Hubner, \& Stanton, 1976), o los pensamientos y sentimientos relacionados con uno mismo (Rosenberg, 1979). Además de por el interés en sí misma, esta variable se ha mostrado esencial

" Dirección postal: Universidad ESAN, Alonso de Molina, 1652, Monterrico Chico, Lima 33, Santiago de Surco,Perú. E-mail: vbustos@esan.edu.pe, oliver@uv.es y laura.galiana@uv.es en el conocimiento del ser humano, estando presente en muchas de las áreas de la psicología, como, por ejemplo, la clínica (Gual et al., 2002; Izquierdo, 2001), la psicología social (Arranz, Yenes, Olabarrieta, \& Martín, 2001; Aznar, 2004; Musitu \& García, 2004), o la psicología evolutiva y de la educación (Rodríguez, Cabanach, Valle, Nuñez, \& González-Pienda, 2004). Más recientemente, el autoconcepto se ha mostrado relevante en el contexto de la psicología positiva, como nuevo acercamiento al estudio de las experiencias y los rasgos positivos (Duckworth, Steen, \& Seligman, 2005), relacionándose positivamente con una mayor satisfacción con la vida (Diener \& Diener, 
2009; Lau \& Leung, 1992; Rey, Extremera, \& Pena, 2011) y con mayor esperanza (Cedeno, Elias, Kelly, \& Chu, 2010; Gilmore, 2014). La conexión entre el autoconcepto y estos dos constructos es clave bajo esta nueva óptica cuyo origen se posiciona en las acciones que llevan al bienestar de las personas, en las cualidades positivas que nos llevan a la felicidad (Seligman \& Csikszentmihalyi, 2000).

Dada su omnipresencia a lo largo de la literatura y la dificultad para su definición, existe un amplio abanico de instrumentos para su medida: medidas observacionales, técnicas proyectivas o medidas de autoevaluación, siendo éstas últimas las más utilizadas (Tomás, Oliver, Galiana, Sancho, \& Lila, 2013). Podemos citar, entre otras, la Escala de Autoestima de Janis y Field (1959), la Escala de Autoestima de Rosenberg (RSES, 1965), el Cuestionario de Auto-Descripción I y sus versiones posteriores (Marsh, 1988) o la Escala Multidimensional del Autoconcepto (Battle, 1992).

En el contexto iberoamericano, aunque existe gran diversidad, destaca por su amplio uso la Escala Multidimensional de Autoconcepto Forma 5 (García \& Musitu, 2014). Este instrumento surgió a partir del modelo teórico de Shavelson et al. (1976), en el que el autoconcepto se caracteriza por su organización jerárquica a partir de una dimensión general. Las dimensiones específicas del autoconcepto, según este instrumento, incluirían el autoconcepto profesional/académico, social, emocional, familiar y físico (García \& Musitu, 2014). Estas dimensiones, aunque relacionadas, se relacionan diferencialmente con distintas áreas del comportamiento humano (García, Musitu, \& Veiga, 2006). Las cinco dimensiones propuestas por los autores, se evalúan mediante 6 ítems que los autores asignaron a priori. Este instrumento permite, pues, medir con un único instrumento las principales dimensiones del autoconcepto (Grandmontagne \& Fernández, 2004).

Las propiedades psicométricas de la Escala Multidimensional Autoconcepto Forma 5 (AF5) se han estudiado en España, tanto en castellano (García \& Musitu, 2014) como en catalán (Malo, Bataller, Casas, Gras, \& González, 2011), así como en Italia (Marchetti, 1997), Brasil (Martínez \& García, 2008; Martínez, García, \& Yubero, 2007; Martínez, Musitu, García, \& Camino, 2003), Portugal (García et al., 2006). En todos estos estudios, la estructura multidimensional se sostiene, además con unas estimaciones de consistencia interna adecuada. García et al. (2006), por ejemplo, informó estimaciones de entre .73 y .88 para las diferentes dimensiones en población española y de entre .76 y .87 en población portuguesa, con mayor consistencia interna para la dimensión del autoconcepto académico/profesional y menores para el autoconcepto físico. Además, recientemente se ha utilizado en otros proyectos de investigación en México, Ecuador, Panamá, Puerto Rico y Venezuela (García \& Musitu, 2014).

En el contexto peruano, sin embargo, no se conocen hasta el momento estudios sobre las propiedades psicométricas (validez, fiabilidad) de dicho instrumento, aunque sí que se ha utilizado en investigaciones previas (Tarazona, 2005). De hecho, precisamente en Perú, el Ministerio de
Salud (2013) lo recoge como uno de los más utilizados en el campo de la salud mental y la psiquiatría. Tal y como señala la American Educational Research Association (AERA), American Psychological Association (APA) y National Council on Measurement in Education (NCME), la validez se refiere al "grado en que la evidencia y la teoría dan apoyo a las interpretaciones de las puntuaciones de las pruebas implicadas para los usos propuestos de un test" (2004, p. 9), es decir, en qué medida se puede confiar de manera legítima en los resultados de un test concreto. Pero estos resultados de medida tienen significado (validez), solo en el contexto del constructo que tienen por objetivo medir (Messick, 1989): la validez no es una propiedad de los instrumentos en sí, sino de las puntuaciones en éstos y de su interpretación (AERA et al., 2004). Por ello, la validez se deberá establecer o evaluar para cada aplicación o interpretación en un contexto diferente de un instrumento de medida, porque cada contexto comportará una interpretación distinta de las puntuaciones (Messick, 1989).

De todo esto se deduce, pues, la necesidad de estudiar el comportamiento de los cuestionarios, en concreto, del AF5, en aquellas poblaciones, como la peruana, en las que no se conocen las bondades psicométricas del instrumento. En consecuencia, el objetivo de este estudio es presentar las propiedades psicométricas de la escala multidimensional Autoconcepto Forma 5 en población peruana, en concreto, en universitarios peruanos.

\section{Método}

\section{Muestra}

La muestra total estuvo compuesta de 527 estudiantes de universidades públicas y privadas peruanas. De estos, el $57.9 \%$ fueron mujeres. En cuanto a la edad, un $29.6 \%$ tenía entre 19 y 20 años, un $38.0 \%$ entre 21 y 22 , un $20.7 \%$ entre 23 y 24 , un $8.2 \%$ entre 25 y 30 y tan solo un $3.5 \%$ tenía más de 30 años. La mediana fue 19.5 años. Finalmente, un $28.7 \%$ estudiaban en el momento del presente trabajo Contabilidad, un $18.4 \%$ Administración, un $15.6 \%$ Administración y Marketing, un 13.5\% Economía, un $7.8 \%$ Psicología Organizacional, un $6.8 \%$ Psicología del Consumidor, un $4.8 \%$ Administración y Finanzas, un $2.5 \%$ Derecho Comercial, un 1.1\% Ingeniería Industrial y el $.8 \%$ restante otras carreras.

\section{Instrumentos}

Además de las preguntas sobre los datos-sociodemográficos expuestos, la encuesta también incluyó, entre otros, los siguientes instrumentos de evaluación:

Escala Autoconcepto Forma 5 (García \& Musitu, 2014). Este instrumento evalúa cinco dimensiones del autoconcepto: académica, social, emocional, familiar y física, con seis ítems cada una. El autoconcepto académico hace referencia a la percepción del desempeño del rol de estudiante; el social, a la percepción del desempeño en las relaciones sociales; el emocional, al estado emocional y la implicación y compromiso con la vida cotidiana; el familiar, a la integración en la familia; y el físico, a la 
percepción del aspecto y la condición física (García \& Musitu, 2014). Los ítems puntúan en una escala tipo Likert que admite 11 posibilidades de respuesta $(0=$ nunca y 10 = siempre), siguiendo las recomendaciones de Cummins y Gullone (2000).

Escala de Esperanza Disposicional (Snyder et al., 1991). La Escala de Esperanza Disposicional parte del modelo desarrollado por Charles R. Snyder y conceptualiza la esperanza como un constructo motivacional, con un fuerte componente cognitivo. Inicialmente Snyder lo conceptualizó como un instrumento de 12 ítems en tres agrupaciones de 4 , para evaluar medios o vías para la esperanza (pathways) acción (agency) y 4 ítems neutros (que pronto cayeron en desuso). La adaptación española, sin embargo, evidenció una estructura unidimensional (Galiana, Oliver, Sancho, \& Tomás, 2015). De esta forma, los
8 ítems, que puntúan en una escala tipo Likert desde 1 (definitivamente falso) hasta 4 (definitivamente verdadero), evalúan un único constructo de esperanza disposicional.

Escala de Satisfacción con la Vida (Diener, Emmons, Larsen, \& Griffin, 1985). La Escala de Satisfacción con la Vida fue desarrollada por Diener y colaboradores (1985), siendo uno de los instrumentos más utilizados en este campo de la investigación (Pavot \& Diener, 2008). Está compuesta por 5 ítems que evalúan un único constructo, el sentimiento general de satisfacción de la persona con su vida como un todo (Diener et al., 1985). Estos cinco ítems tienen escala tipo Likert de 5 anclajes, desde 1 (totalmente en desacuerdo) hasta 5 (totalmente de acuerdo).

Los estadísticos descriptivos para estas variables, así como sus estimaciones de consistencia interna, pueden observarse en la Tabla 1.

Tabla 1

Consistencia Interna y Estadísticos Descriptivos de las Variables Utilizadas en el Estudio

\begin{tabular}{lccc}
\hline & $M$ & $S D$ & $\alpha$ \\
\hline Esperanza disposicional & 3.25 & .36 & .75 \\
Satisfacción con la vida & 3.54 & .76 & .78 \\
Autoconcepto académico & 6.97 & 1.39 & .81 \\
Autoconcepto social & 7.10 & 1.54 & .73 \\
Autoconcepto emocional & 5.14 & 2.03 & .82 \\
Autoconcepto familiar & 7.65 & 1.65 & .76 \\
Autoconcepto físico & 6.67 & 1.70 & .75 \\
\hline
\end{tabular}

Notas. $M=$ media; $S D=$ desviación típica.

\section{Diseño}

El presente trabajo sigue un diseño de encuesta transversal. Una vez obtenidas las autorizaciones de los comités de ética correspondientes, se llevaron a cabo encuestas de lápiz y papel en centros de educación superior en Perú públicos y privados, en un mismo momento temporal. La participación de los estudiantes fue totalmente voluntaria e informada, con consentimiento informado.

Para llevar a cabo dichas entrevistas, entrevistadores formados se desplazaron a los centros en horario de clases, realizando el pase en aulas de las instituciones educativas participantes. Se tardaba aproximadamente 30 minutos en completar la encuesta.

\section{Análisis de Datos}

En primer lugar, se procedió al estudio de la validez de constructo de la medida. Para ello, especificó, estimó y evaluó un modelo de ecuaciones estructurales, en concreto, un análisis factorial confirmatorio (AFC), en el que se probó la estructura original propuesta por los autores. En este caso, el AFC que se especificó hipotetizaba 5 factores correspondientes a las dimensiones del autoconcepto propuestas por los autores de la escala original: autoconcepto académico, social, emocional, familiar y físico, correlacionados entre sí (García \& Musitu, 2014). Dada la naturaleza de los datos, el método de estimación empleado fue máxima verosimilitud con correcciones robustas para los errores estándar, sobre la matriz de correlaciones policóricas (Finney \& DiStefano, 2006).

La adecuación o plausibilidad del modelo se evaluó mediante diversos criterios, tal y como recomienda la literatura (Byrne, 2012): (a) el estadístico chi-cuadrado $\left(\chi^{2}\right)$, el índice de ajuste original de los modelos de ecuaciones estructurales (Wang \& Wang, 2012), con correcciones robustas, que evalúa la magnitud de la discrepancia entre las matrices de varianzas y de covarianzas de la muestra y las del modelo estimado, aunque sufre de diversas limitaciones (Kline, 2011); (b) el Comparative Fit Index (CFI), que es indicativo de un buen ajuste cuando tiene valores superiores a $.90 \mathrm{e}$, idealmente, superiores a .95 (Hu \& Bentler, 1999); (c) Bentler-Bonnet Non-Normed Fit Index (BBNFI), con misma interpretación que CFI; (d) el Bollen's Fit Index (IFI), también con puntos de corte de .90 para indicar buen ajuste; y (e) el Root Mean Square 
Bustos,V., Oliver, A. \& Galiana, L. (2015). Validación del Autoconcepto Forma 5 en Universitarios Peruanos: Una Herramienta para la Psicología Positiva.

Error of Approximation (RMSEA), con valores iguales o inferiores a .08 como indicadores de un buen ajuste del modelo (Hu \& Bentler, 1999).

En segundo lugar, y con el objetivo de estudiar la fiabilidad de las medidas, se calcularon estadísticos descriptivos para los ítems de las diferentes escalas (medias y desviaciones típicas) y el alfa de Cronbach. El alfa es el índice de fiabilidad más ampliamente utilizado y conocido por la comunidad científica para evaluar la fiabilidad de tests y escalas, con valores de .70 a .79 considerados moderados y de .80 o superiores interpretados como alta fiabilidad (Cicchetti, 1994). Otras estimaciones de fiabilidad calculadas incluyeron la homogeneidad de los ítems.

Finalmente, para evaluar la validez criterial, se utilizaron correlaciones de Pearson con dos medidas tradicionalmente relacionadas con el autoconcepto: la esperanza y la satisfacción con la vida.

Los análisis se realizaron con los paquetes estadísticos EQS 6.1 y SPSS 20.

\section{Resultados}

\section{Validez de Constructo}

$\mathrm{El}$ análisis factorial confirmatorio realizado para evaluar la validez de constructo de la Escala Autoconcepto Forma 5 ofreció unos índices de ajuste adecuados, $\chi_{S B}^{2}$ $(395)=1484.46, p<.01 ; \mathrm{CFI}=.93 ; \mathrm{BBNFI}=.92 ; \mathrm{IFI}=$ .93 ; y RMSEA $=.07$ [intervalo de confianza al $90 \%$ : .07.08]. El ajuste analítico del modelo, también fue óptimo, tal y como se puede observar en la Figura 1, con saturaciones factoriales con valores entre .21 (ítem 22, "Me cuesta hablar con desconocidos/as") y .84 (ítem 29, "Me siento querido/a por mis padres"). Las correlaciones también fueron en la dirección adecuada, siendo todas positivas y estadísticamente significativas $(p<.01$; Tabla 2$)$.

Figura 1. Saturaciones factoriales del modelo de ecuaciones estructurales propuesto para la Escala Autoconcepto Forma 5. Notas. Todas las cargas factoriales fueron estadísticamente significativas $(p<.05)$. Los valores de las correlaciones pueden observarse en la Tabla 1. 


\section{Fiabilidad}

La consistencia interna fue adecuada, con alfas que oscilaron entre .75 para el autoconcepto físico y .82 para el autoconcepto emocional (ver Tabla 1). Información sobre la consistencia interna de los ítems puede observarse en la Tabla 3.

\section{Validez Criterial}

Por lo que respecta a las relaciones de las dimensiones del autoconcepto con la esperanza y la satisfacción con la vida, y como se ve en la Tabla 4, fueron todas positivas y estadísticamente significativas, en la línea de lo apuntado previamente en la literatura (Cedeno et al., 2010; Diener \& Diener, 2009; Gilmore, 2014; Rey et al., 2011).

Tabla 2

Correlaciones de los Factores de la Escala Autoconcepto Forma 5

\begin{tabular}{cccccc}
\hline & A. Académico & A. Social & A. Emocional & A. Familiar & A. Físico \\
\hline A. Académico & 1 & -- & -- & -- & -- \\
A. Social & $.63^{*}$ & 1 & -- & -- & -- \\
A. Emocional & $.31^{*}$ & $.26^{*}$ & 1 & -- & -- \\
A. Familiar & $.49^{*}$ & $.67^{*}$ & $.26^{*}$ & 1 & -- \\
A. Físico & $.69^{*}$ & $.68^{*}$ & $.31^{*}$ & $.66^{*}$ & 1 \\
\hline
\end{tabular}

Notas. A. Académico = Autoconcepto académico; A. Social = Autoconcepto social; A. Emocional = Autoconcepto emocional; A. Familiar $=$ Autoconcepto familiar; A. Físico $=$ Autoconcepto físico.

$* p<.01$.

Tabla 3

Estadísticos Descriptivos y Homogeneidad de los Ítems del Autoconcepto Forma 5

\begin{tabular}{|c|c|c|c|c|c|c|c|c|c|c|c|c|c|c|c|c|c|c|c|}
\hline \multicolumn{4}{|c|}{ A. Académico } & \multicolumn{4}{|c|}{ A. Social } & \multicolumn{4}{|c|}{ A. Emocional } & \multicolumn{4}{|c|}{ A. Familiar } & \multicolumn{4}{|c|}{ A. Físico } \\
\hline Ítem & $M$ & $S D$ & $\alpha_{i i}$ & Ítem & $M$ & $S D$ & $\alpha_{i i}$ & Ítem & $M$ & $S D$ & $\alpha_{i i}$ & Ítem & $M$ & $S D$ & $\alpha_{i i}$ & Ítem & $M$ & $S D$ & $\alpha_{i i}$ \\
\hline 1 & 7.54 & 1.47 & .78 & I 2 & 7.62 & 1.99 & .64 & $\mathrm{I} 3$ & 3.63 & 2.43 & .82 & I 4 & 5.94 & 2.98 & .74 & I 5 & 6.41 & 2.36 & .73 \\
\hline I6 & 6.97 & 1.81 & .74 & I7 & 7.92 & 1.72 & .66 & I8 & 4.46 & 2.68 & .78 & I9 & 7.67 & 2.20 & .71 & I 10 & 5.83 & 3.20 & .70 \\
\hline I11 & 6.30 & 2.66 & .86 & $\mathrm{I} 12$ & 6.68 & 3.00 & .68 & I13 & 6.18 & 2.84 & .79 & $\mathrm{I} 14$ & 7.98 & 2.65 & .72 & I 15 & 6.46 & 2.33 & .72 \\
\hline I16 & 6.80 & 2.02 & .75 & $\mathrm{I} 17$ & 7.86 & 1.96 & .69 & I18 & 5.60 & 2.93 & .79 & I19 & 8.05 & 2.72 & .74 & I 20 & 7.68 & 2.19 & .71 \\
\hline $\mathrm{I} 21$ & 7.39 & 1.70 & .78 & $\mathrm{I} 22$ & 5.23 & 3.07 & .77 & $\mathrm{I} 23$ & 5.11 & 2.81 & .79 & $\mathrm{I} 24$ & 7.74 & 2.23 & .69 & I 25 & 6.15 & 3.01 & .68 \\
\hline $\mathrm{I} 26$ & 6.91 & 1.82 & .74 & I27 & 7.27 & 2.14 & .67 & $\mathrm{I} 28$ & 5.98 & 2.83 & .78 & I 29 & 8.55 & 1.82 & .69 & I 30 & 7.42 & 2.08 & .71 \\
\hline
\end{tabular}

Notas. $M=$ media; $S D=$ desviación típica; $\alpha_{i i}=$ alfa si se elimina el elemento.

Tabla 4

Correlaciones entre las Dimensiones del Autoconcepto, la Esperanza y la Satisfacción con la Vida

\begin{tabular}{ccc}
\hline & Esperanza & Satisfacción \\
\hline A. Académico & $.374^{*}$ & $.331^{*}$ \\
A. Social & $.341^{*}$ & $.224^{*}$ \\
A. Emocional & $.251^{*}$ & $.194^{*}$ \\
A. Familiar & $.246^{*}$ & $.337^{*}$ \\
A. Físico & $.308^{*}$ & $.308^{*}$ \\
\hline
\end{tabular}

$* p<.01$ 
Bustos,V., Oliver, A. \& Galiana, L. (2015). Validación del Autoconcepto Forma 5 en Universitarios Peruanos: Una Herramienta para la Psicología Positiva.

\section{Discusión}

Dado el papel relevante del autoconcepto a lo largo de la psicología (James, 1890; Shavelson et al., 1976), es pues necesario contar con instrumentos para su medida, instrumentos que han probar sus bondades psicométricas para la aplicación o interpretación en cada contexto (AERA et al., 2004; Messick, 1989). De esta forma, el objetivo de este estudio ha sido aportar evidencias de validez y fiabilidad de la escala multidimensional Autoconcepto Forma 5 en una muestra de estudiantes universitarios peruana, siendo éste, hasta donde conocemos, el primer trabajo llevado a cabo a tal fin.

Para conseguir dicho objetivo, se especificó, estimó y evaluó, en primer lugar, un modelo de ecuaciones estructurales con la estructura original propuesta por los autores. Los resultados de este análisis fueron adecuados, tanto a nivel general como analítico, y van en la línea de la literatura consultada (García, Musitu, Riquelme, \& Riquelme, 2011; García et al., 2006). En este sentido, todas las cargas factoriales, así como las correlaciones entre los factores, fueron estadísticamente significativas. Tan solo el ítem 22 tuvo una saturación por debajo de lo esperado, aunque se retuvo en el modelo por motivos sustantivos y la adecuada representatividad del constructo (Messick, 1989). Por lo que respecta a las relaciones entre las dimensiones del autoconcepto, se encontró apoyo a lo detectado en estudios previos (García et al., 2011): el social y el físico mostraron las correlaciones más elevadas con el resto de dimensiones, frente al emocional, que arrojó las de menor tamaño. Futuras investigaciones deberían investigar en profundidad este resultado, esta mayor independencia del autoconcepto emocional, frente al autoconcepto social y físico, que tendrían mayor peso en el autoconcepto general.

Los resultados de consistencia interna también fueron adecuados, con estimaciones de alfa de Cronbach muy similares a la de estudios previos (véase, por ejemplo, García et al., 2011; García et al., 2006; Malo et al., 2011). Tan solo los ítems 11 y 22 mostraron menor consistencia, aunque ambos habían saturado de forma estadísticamente significativa en el modelo. En rangos similares encontramos las estimaciones ofrecidas por García et al. (2006) para población española y portuguesa.

Finalmente, en cuanto a la validez criterial, todas las relaciones entre las dimensiones del autoconcepto, la esperanza disposicional y la satisfacción con la vida fueron estadísticamente significativas y positivas, tal y como se ha recogido en trabajos previos (Cedeno et al., 2010; Diener \& Diener, 2009; Gilmore, 2014; Rey et al., 2011). Por ejemplo, en un trabajo reciente, Rey et al. (2011) encontraron una correlación de .59 entre la autoestima general y la satisfacción con la vida. Valores similares se han recogido para la relación entre el autoconcepto y la esperanza disposicional (.58, por ejemplo, en el estudio de Gilmore, 2014), aunque también de menor magnitud, como en el caso de Cedeno et al. (2010), en el que el autoconcepto predijo un $9 \%$ de la esperanza. De esta forma, fomentar el autoconcepto de los universitarios peruanos parece clave para garantizar su disposición hacia la consecución de metas (esperanza disposicional) y su bienestar (satisfacción con la vida).

En conclusión, los resultados de la presente investigación ofrecen evidencia de la estructura de cinco dimensiones de la escala multidimensional AF5, con adecuadas estimaciones de consistencia y validez criterial en estudiantes universitarios peruanos. Como fortalezas de esta investigación destacamos, de un lado, la utilidad que ha mostrado la AF5 en investigaciones previas en distintas poblaciones, ofreciendo pues un instrumento de amplio uso con apropiadas propiedades psicométricas en un nuevo contexto. De otro, la consistencia de los resultados con la literatura previa, tanto en lo que respecta a dimensionalidad, como a fiabilidad o validez criterial. $\mathrm{Y}$, finalmente, el hecho de que el instrumento validado evalúe el constructo del autoconcepto, permitirá en futuras investigaciones en el contexto peruano no sólo conocer las garantías psicométricas de sus aplicación (una necesidad que identificaban recientemente los propios informes del Ministerio de Salud en 2013), sino ahondar más en el nuevo dibujo que ha supuesto la psicología positiva dentro de la psicología tradicional. En este sentido, las relaciones que el autoconcepto ha mostrado con la satisfacción con la vida y la esperanza, algunas de las cualidades positivas que conforman la felicidad y la "buena vida", tal y como se entiende desde la Psicología Positiva (Seligman \& Csikszentmihalyi, 2000), hacen que se trate de un elemento clave en la vida de los estudiantes universitarios peruanos.

En cuanto a las limitaciones, cabe destacar el diseño transversal, que no permitió obtener medidas de fiabilidad temporal, o la naturaleza incidental de la muestra, que por otra parte facilita la comparación de sus resultados, dado que la mayoría de estudios de validación de la AF5 realizados hasta el momento se habían llevado a cabo también en estudiantes recogidos de forma no probabilística.

\section{Referencias}

American Educational Research Association, American Psychological Association, \& National Council on Measurement in Education. (2004). Standards for educational and psychological testing ( $2^{\text {nd }}$ ed.). Washington, DC: American Educational Research Association.

Arranz, E., Yenes, F., Olabarrieta, F., \& Martín, J. L. (2001). Relaciones entre hermanos y desarrollo psicológico en escolares. Infancia y Aprendizaje, 24, 361-377. doi:10.1174/021037001316949275

Aznar, M. P. M. (2004). Impacto de la violencia doméstica en la salud de la mujer maltratada. Psicothema, 16, 397-401.

Battle, J. (1992). Culture-free Self-esteem Inventories. Austin, TX: PRO-ED.

Byrne, B. M. (2012). Structural equation modeling with Mplus: Basic concepts, applications, and programming. New York: Routledge. 
Cedeno, L. A., Elias, M. J., Kelly, S., \& Chu, B. C. (2010) School violence, adjustment, and the influence of hope on low-income, African American youth. American Journal of Orthopsychiatry, 80, 213-226. doi:10.1111/j.19390025.2010.01025.x

Cicchetti, D. V. (1994). Guidelines, criteria, and rules of thumb for evaluating normed and standardized assessment instruments in psychology. Psychological Assessment, 6, 284-290. doi:10.1037/1040-3590.6.4.284

Cummins, R. A., \& Gullone, E. (2000). Why we should not use 5-point Likert scales: The case for subjective quality of life measurement. In Proceedings, Second International Conference on Quality of Life in Cities (pp.74-93). Singapore, Republic of Singapore: National University of Singapore.

Diener, E., \& Diener, M. (2009). Cross-cultural correlates of life satisfaction and self-esteem. In E. Diener (Ed.), Culture and well-being (pp. 71-91). Amsterdam, Holland: Springer.

Diener, E., Emmons, R. A., Larsen, R. J., \& Griffin, S. (1985). The Satisfaction with Life Scale. Journal of Personality Assessment, 49, 71-75. doi:10.1207/s15327752jpa4901_13

Duckworth, A. L., Steen, T. A., \& Seligman, M. E. P. (2005). Positive psychology in clinical practice. Annual Review of Clinical Psychology, 1, 629-651. doi:10.1146/annurev. clinpsy.1.102803.144154

Finney, S. J., \& DiStefano, C. (2006). Non-normal and categorical data in SEM. In G. R. Hancock \& R. O. Mueller (Eds.), Structural equation modeling: A second course (pp. 269-314). Greenwich, CO: Information Age.

Galiana, G., Oliver, A., Sancho, P., \& Tomás, J. M. (2015). Dimensionality and validation of the Dispositional Hope Scale in a Spanish sample. Social Indicators Research, 120, 297-308. doi:10.1007/s11205-014-0582-1

García, J. F., \& Musitu, G. (2014). AF5: Autoconcepto forma 5. Madrid, España: TEA.

García, J. F., Musitu, G., Riquelme, E., \& Riquelme, P. (2011). A confirmatory factor analysis of the "Autoconcepto Forma 5" questionnaire in young adults from Spain and Chile. Spanish Journal of Psychology, 14(2), 648-658. doi:10.5209/ rev_SJOP.2011.v14.n2.13

García, J. F., Musitu, G., \& Veiga, F. (2006). Autoconcepto en adultos de España y Portugal. Psicothema, 18(3), 551-556.

Gilmore, A. L. (2014). A cultural examination of hardiness: Associations with self-esteem, wisdom, hope, and coping-efficacy [Monograph]. Retrieved from http:// digitalcommons.georgiasouthern.edu/cgi/viewcontent. cgi? article $=1004 \&$ context $=$ honors-theses

Grandmontagne, A. G., \& Fernández, A. R. (2004). Eating disorders, sport practice and physical self-concept in adolescents. Actas Españolas de Psiquiatría, 32, 29-36.

Gual, P., Pérez, M., Martinez, M. A., Lahortiga, F., Irala, J., \& Cervera, S. (2002). Self-esteem, personality and eating disorders: Baseline assessment of a prospective populationbased cohort. International Journal of Eating Disorders, 31, 261-273. doi:10.1002/eat.10040

Hu, L. T., \& Bentler, P. M. (1999). Cutoff criteria for fit indexes in covariance structure analysis: Conventional criteria versus new alternatives. Structural Equation Modeling, 6, 1-55. doi:10.1080/10705519909540118

Izquierdo, F. M. (2001). Un programa de prevención con hijos de alcohólicos. Anales de Psiquiatría, 17, 313-318.

James, W. (1890). Principles of psychology. New York: Holt.
Janis, I. L., \& Field, P. B. (1959). A behavioral assessment of persuasibility: Consistence of individual differences. In C. I. Hovland \& I. L. Janis (Eds.), Personality and persuasibility (pp. 259-268). New Haven, CT: Yale University Press.

Kline, R. B. (2011). Principles and practice of structural equation modeling ( $3^{\text {rd }}$ ed.). New York: Guilford Press.

Lau, S., \& Leung, K. (1992). Relations with parents and school and Chinese self-concept, delinquency and academic performance. British Journal of Educational Psychology, 62, 193-202.

Malo, S., Bataller, S., Casas, F., Gras, M. E., \& González, M. (2011). Análisis psicométrico de la escala multidimensional de autoconcepto AF5 en una muestra de adolescentes y adultos de Cataluña. Psicothema, 23(4), 871-878.

Marchetti, B. (1997). Concetto di se'relazioni familiari e valori (Tesis de Licenciatura en Psicología Social no publicada). Universita degli Studi di Bologna, Italia.

Marsh, H. W. (1988). Self-description Questionnaire-I. San Antonio, TX: Psychological Corporation.

Martínez, I., \& García, J. F. (2008). Internalization of values and self-esteem among Brazilian teenagers from authoritative, indulgent, authoritarian, and neglectful home. Adolescence, 43(169), 13-29.

Martínez, I., García, J. F., \& Yubero, S. (2007). Parenting styles and adolescents' self-esteem in Brazil. Psychological Reports, 100, 731-745. doi:10.2466/PR0.100.3.731-745

Martínez, I., Musitu, G., García, J. F., \& Camino, L. (2003). Un análisis intercultural de los efectos de la socialización familiar en el autoconcepto: España y Brasil. Psicología, Educaçâo e Cultura, 7, 239-258.

Messick, S. (1989). Validity. In R. L. Linn (Ed.), Educational measurement (pp. 13-103). New York: Macmillan.

Ministerio de Salud. (2013). Actualización de la base de datos de instrumentos de evaluación de salud mental y psiquiatría 2012. Lima, Perú: Autor.

Musitu, G., \& García, J. F. (2004). Consequences of the family socialization in the Spanish culture. Psicothema, 16, 288-293.

Pavot, W., \& Diener, E. (2008). The satisfaction with life scale and the emerging construct of life satisfaction. The Journal of Positive Psychology, 3(2), 137-152. doi:10.1080/17439760701756946

Rey, L., Extremera, N., \& Pena, M. (2011). Perceived emotional intelligence, self-esteem and life satisfaction in adolescents. Psychosocial Intervention, 20, 227-234. doi:10.5093/in2011v20n2a10

Rodríguez, S., Cabanach, R. G., Valle, A., Nuñez, J. C., \& González-Pienda, J. A. (2004). Differences in use of selfhandicapping and defensive pessimism and its relation with achievement goals, self-esteem and self-regulation strategies. Psicothema, 16, 625-631.

Rosenberg, M. (1965). Society and the adolescent self-image. Princeton, NJ: Princeton University Press.

Rosenberg, M. (1979). Conceiving the self. New York: Basic Books.

Seligman, M. E. P., \& Csikszentmihalyi, M. (2000). Positive psychology. An introduction. American Psychologist, 55, 5-14. doi:10.1037/0003-066X.55.1.5

Shavelson, J., Hubner, J. J., \& Stanton, G. C. (1976). Self-concept: Validation of construct interpretations. Review of Educational Research, 46, 407-442. doi:10.3102/00346543046003407 
Bustos,V., Oliver, A. \& Galiana, L. (2015). Validación del Autoconcepto Forma 5 en Universitarios Peruanos: Una Herramienta para la Psicología Positiva.

Snyder, C. R., Harris, C., Anderson, J. R., Holleran, S. A., Irving, L. M., Sigmon, S. T., ...Harney, P. (1991). The will and the ways: Development and validation of an individual-differences measure of hope. Journal of Personality and Social Psychology, 60, 570-585. doi:10.1037/0022-3514.60.4.570

Tarazona, D. (2005). Autoestima, satisfacción con la vida y condiciones de habitabilidad en adolescentes estudiantes de quinto año de media. Un estudio factorial según pobreza y sexo. Revista IIPS, 8, 57-65.

Tomás, J. M., Oliver, A., Galiana, L., Sancho, P., \& Lila, M. (2013). Explaining method effects associated with negatively worded items in trait and state global and domain-specific Self-esteem Scales. Structural Equation Modeling, 20, 299 313. doi:10.1080/10705511.2013.769394

Wang, J., \& Wang, X. (2012). Structural equation modeling. Applications using Mplus. West Sussex, UK: Wiley. 